\title{
Eustachian Tube Opening Measurement by Sonotubometry Using Perfect Sequences for Healthy Adults
}

\author{
Vilma Beleskiene ${ }^{1,2} \cdot$ Eugenijus Lesinskas $^{1,2} \cdot$ Vaida Januskiene $^{1,2} \cdot$ Kristina Daunoraviciene $^{3} \cdot$ Darius Rauba $^{1,2}$ \\ Justinas Ivaska ${ }^{1,2}$
}

${ }^{I}$ Clinic of Ear, Nose, Throat and Eye Diseases, Faculty of Medicine, Vilnius University, Vilnius; ${ }^{2}$ Center of Ear, Nose and Throat Diseases, Vilnius University Hospital Santariskiu Clinics, Vilnius, ${ }^{3}$ Faculty of Mechanics, Vilnius Gediminas Technical University, Vilnius, Lithuania

Objectives. The aim of this study was to establish the rate variation of sonotubometric measurements using a specific broadband class of signals, the so-called perfect sequences (PSEQ) among healthy adults and to identify an optimal and technically simple test to provoke Eustachian tube (ET) openings.

Methods. Sonotubometry was performed on 105 healthy adult subjects. Three different consecutive maneuvers were performed for ET opening: dry swallowing, water swallowing (a small [2 $\mathrm{mL}]$ and a large [5 $\mathrm{mL}]$ water bolus). Values of the amplitude and duration of each measured ET opening were calculated.

Results. A total of 6,300 measurements were performed. Sonotubometric ET openings were detected for all subjects but not for each measurement. In 6,180 of 6,300 measurements (98.1\%), objective ET openings were registered. In 11 of 105 subjects $(10.5 \%)$ at least one sonotubometric ET opening was not detected. The mean ET opening duration time and the mean sound amplitude similar for all performed test and were $270(\mathrm{SD}, \pm 96) \mathrm{msec}, 13.48$ (SD, \pm 6.57$)$ $\mathrm{dB}$.

Conclusion. Sonotubometry based on PSEQ stimuli is a reliable methodology to assess the ET opening function in healthy subjects. Mean ET opening duration time and the mean sound wave amplitude performed similarly in all analysed tests, hence it might be concluded that dry (saliva) and water swallowing are reliable sonotubometric maneuvers and may be used when examining ET opening function. The size of a sip during water swallowing does not affect the sonotubometry result. All maneuvers can be equally used as the optimal test, and water swallow is most comfortable for the subject.

Keywords. Eustachian Tube; Acoustic Impedance Tests; Adult

\section{INTRODUCTION}

Eustachian tube (ET) dysfunction is most common in children and almost $40 \%$ of children under 10 years of age experience temporary ET dysfunction [1]. In adults this condition is rela-

- Received April 24, 2015

Revised June 25, 2015

Accepted July 29, 2015

- Corresponding author: Vilma Beleskiene

Clinic of Ear, Nose, Throat and Eye Diseases, Faculty of Medicine,Vilnius

University, Santariskiu 2, Vilnius, Lithuania

Tel: +370-65201000, Fax: +370-52365270

E-mail: vilmabeleskiene@gmail.com tively rare and it is believed that prevalence of ET dysfunction ranges from $0.9 \%$ to $5 \%[2,3]$. An impaired ET is closely linked with such middle ear diseases as chronic otitis media, tympanic membrane perforation, secretory otitis, or cholesteatoma [4,5], yet the origin of the pathology and the role of the ET in the pathogenesis remain unclear to this date. An attic retraction pocket and cholesteatoma can develop in subjects with a normally functioning ET due to selective epitympanic dysventilation syndrome that can be caused due to blocked isthmus [6]. However, it is often unclear when these pathogenetic conditions are essentially caused by ET dysfunction and when they are the result of mucosal gas production and metabolism in the im-

Copyright @ 2016 by Korean Society of Otorhinolaryngology-Head and Neck Surgery.

This is an open-access article distributed under the terms of the Creative Commons Attribution Non-Commercial License (http://creativecommons.org/licenses/by-nc/4.0)

which permits unrestricted non-commercial use, distribution, and reproduction in any medium, provided the original work is properly cited. 
paired middle ear. Objective high-quality and technically simple tests for ET function can be clinically essential in order to better understand the pathogenesis and stages of and to determine the treatment for these diseases. Knowledge about The ET function would be beneficial in planning surgical treatment of the middle ear and inferring outcomes of such treatment. Emerging methods in surgical treatments urge the necessity of objective information on ET function for patient selection and postoperative evaluation of results.

In recent years, a number of clinical articles have presented data on a range of distinct ET test methods like sonotubometry, tubomanometry, manometric tests, tensometry, computed tomography, and magnetic resonance imaging, however there is no consensus on their application and use in daily practice [7]. Most testing methods are subjective and nonspecific, likewise objective methods are insufficiently standardised and they poorly correlate with the clinical picture or are nonphysiological, therefore employed only under certain pathological conditions (e.g., manometric methods are suitable in patients with a tympanic membrane perforation) [8-10]. Among the many studies, there is no 'golden standard' which could be widely used and serve as a benchmark to all. In order to clarify the pathogenesis of diseases and ET function, it is essential to obtain a reliable test which presents quantitative assessment of the function.

Sonotubometry is an objective method to test ET ventilation function by measuring sound and its intensity changes during ET openings. The sonotubometry principle was first introduced by Politzer in 1869 [11]. Even though a number of clinical trials have been carried out to evaluate the effectiveness of the test and sonotubometry is rightly recognized as an objective and physiological test of ET function, in clinical practice it has not become widespread and it is not used. The measurements obtained can sometimes be interfered with due to noise pollution from the nasopharynx because of saliva movement during swallowing; therefore artefacts are recorded. In other cases, additional curves of unknown origin may be generated and they are difficult to interpret [12-16]. When performing different maneuvers during the course of measurement (dry swallowing, water swallowing, Toynbee maneuver, yawning), the spectrum of results differ quite a lot. Therefore, the published data presents inconsistent results of sonotubometry (range, 50\% to $96 \%$ ) $[17,18]$. One of the possible drawbacks of sonotubometry is that the inconsistent results may by caused by the properties of the acoustic signal itself (commonly the test uses an $8-\mathrm{kHz}$ signal) [19]. Recently, for sonotubometric examination, a specific broadband class of signals, the so-called perfect sequences (PSEQ) was offered and successfully proved results of objective ET openings [18,19]. It can be assumed that the use of PSEQ signal may improve results and improve the validity and reliability of the test [19].

Having used PSEQ and gauged test results from a larger num- ber of healthy subjects, in the present study we aim to establish the rate variation of sonotubometric measurements among healthy adults and to identify an optimal and technically simple test to provoke ET openings.

\section{MATERIALS AND METHODS}

The work was carried out in the Centre of Otorhinolaryngology (ENT) of the Vilnius University Hospital Santariskiu Clinic in 2014.

\section{Subjects}

The study involved 105 otologically healthy adult individuals with no objective ear and nasal pathology data and no record of head and neck operations. The persons' data was collected in anamnesis, and all individuals were subjected to otoscopy, rinoscopy, tympanometry and the Valsalva maneuver. The study included subjects who at the time of the examination did not show any pathological changes, had A type tympanogram and performed positive Valsalva and swallowing maneuvers subjectively. Every person signed an Informed Consent Form to participate in the test. The study was approved by Vilnius Regional Biomedical Research Ethics Committee (No. 158200-13-575-170).

\section{Sonotubometry device}

Technologically, sonotubometry devices come in many variations, some being produced on an industrial scale. In the current study the sonotubometry technology with PSEQ stimuli was employed according to Asenov et al. [18] and Di Martino et al. [19]. With consent and permission of the authors, the a sonotubometry device was constructed by the authors of this study. The system was connected to a computer with the SonoTube software installed. For acoustic stimulation, the external sound card (FireBox PreSonus FIREBOX Inc., Baton Rouge, LO, USA) was used. To send stimulus signals, professional headphones (Shure E3, Heilbronn, Germany; frequency response from 25 $\mathrm{Hz}$ to $18.5 \mathrm{kHz}$ ) were tuned. The headphones were attached to a specially adapted nozzle which could be inserted into the nasal vestibule. For pervasive signals, the sound had an intensity level of $71 \mathrm{~dB}$. To record sound, a miniature microphone (KE 4, Sennheiser, Wedemark, Germany; frequency response from 20 $\mathrm{Hz}$ to $20 \mathrm{kHz}$ ) was used. In order to capture the sound energy transmitted from the speaker to the microphone, a sonotubogram was drawn. As the acoustic stimulus signal, a novel specific broadband class of signals, the so-called PSEQ were used. Being periodic and deterministic random noise signals, PSEQ signals have an ideally have a flat spectrum. With PSEQ signals, all frequency components $(0-24 \mathrm{kHz})$ are stimulated equally during every period.

\section{Measurement procedure}

The testing was conducted with the subject sitting in a quiet 


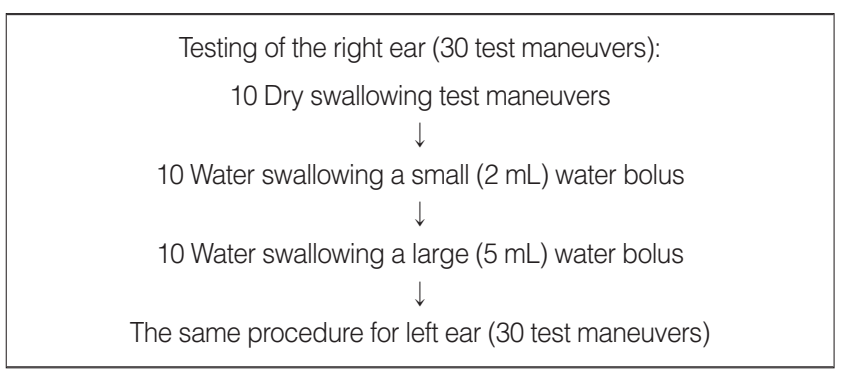

Fig. 1. Sonotubometry measurement procedure.

room. Before the examination, ear wax was removed from the external ear and the subject was induced into the test procedure. Headphones with a special nozzle were placed into the nostril on the opposite side of the investigative ET. Fifteen-second long sounds were produced for each measurement cycle at a sound intensity pressure level of $71 \mathrm{~dB}$. A miniature microphone was inserted into the outer ear canal and it recorded signals transmitted from nose to ear. After measuring one ear, the testing sides were switched.Three different consecutive maneuvers were performed: dry swallowing, water swallowing with a small $(2 \mathrm{~mL})$ water bolus and water swallowing with a large $(5 \mathrm{~mL})$ water bolus. Ten cups with the $2-\mathrm{mL}$ water bolus and 10 cups with the $5-\mathrm{mL}$ water bolus, were presented to the individual for the water swallowing maneuver, in order to swallow equal size sips of water. In order to measure each maneuver, the subjects were instructed to swallow 10 times. The tests were repeated until the subject swallowed 10 times for each maneuver. Consecutively, each subject performed 60 swallowing maneuvers, with 30 for each ear. Simplified measurement procedure is presented in Fig. 1. As the quality of the research may be conditioned by a number of factors affecting the nature of the sound, or on the patient's ability to correctly perform an intricate and demanding series of 60 test maneuvers, in cases of obviously visible artefacts or when the sonotubograms did not record, the test was repeated.

\section{Measurement}

Each measurement was recorded digitally and presented on a computer screen as a diagram (sonotubogram). On the x-axis, time was registered in milliseconds and on the y-axis, the ordinate change in the sound intensity in the outer ear canal was measured using a logarithmic $\mathrm{dB}$ scale. Each sonotubogram was analyzed and the following factors were taken into consideration: the number of ET openings produced against the number of tests maneuvers performed, the amplitude and duration of each registered opening, and the form of the resulting curve. An amplitude of $>5 \mathrm{~dB}$ was required to classify a curve as an opening of the ET. Values of the amplitude and duration of each measured ET opening were calculated. The curves were classified into 1 of 4 different forms: (1) spikes with a sharp curve rise and a descent and including one clear peak, (2) double-peaks when the crest curve has a double peak, (3) descending curves with a wider base and going downward gradually, and (4) plateau curves with a broad base and going downward suddenly. All ears were analyzed as separate independent objects.

\section{Statistical analysis}

One-way analysis of variance was used for comparison of sound intensity values in different sides of the ear. A simple chi-square test to compare 2 proportions was used for comparison of values between different measurements. For comparisons of measurements in patient groups, adequatettests were calculated. All statistical analyses were performed with MATLAB R2011b (MathWorks Inc., Natick, MA, USA).

\section{RESULTS}

The testing was conducted on 105 individuals (33 females [31.4\%] and 72 males [68.6\%]). All of them were able to complete the test. The average age of the subjects was 26.03 (SD, \pm 6.20) (range, 19 to 53 years). A total of 60 measurements were performed on each individual ( 3 test maneuvers, 10 swallowing events, 2 ears per individual). In total, 6,300 measurements were performed. Most patients were easily able to perform the test maneuvers every 1-3 seconds. When subject was not able to perform 10 test maneuvers during 15 -second long sound session, it was repeated until 10 swallowing test maneuvers was performed.

For the majority of the patients, the test detected clear ET openings in all 10 sonotubometric measurements induced by both dry and water swallowing meanuvers. In 11 of 105 subjects $(10.5 \%)$ at least one sonotubometric ET opening was not detected. In 6 patients, at least one ET opening was not recorded in both ears, while in 5 patients no ET opening was detected in one of the ears and all 10 openings were captured in the other ear. In 4 of these patients (3.8\%), no opening was recorded in one of the ears. For most of these patients either obvious artifacts were obtained, or the response, though detected, did not reach the provided $5-\mathrm{dB}$ limit. Therefore, the test was repeated, considering that patients possibly failed to perform the relatively intricate requirements of the test correctly and efficiently at once, especially during dry swallowing. During the repetition test, fewer artifacts and failed ET openings were detected, and all patients recorded at least one ET opening. Finally, sonotubometric ET openings were detected for all subjects but not for each measurement.

Using this sonotubometry methodology, in 6,180 of 6,300 measurements (98.1\%) objective ET openings were registered, which qualified them as valid and corresponding to the criteria. In 7 of 105 subjects (6.7\%), at least one sonotubometric ET opening was not detected. In 3 of these subjects, at least one ET opening was not recorded in both ears, while in 4 subjects the ET opening was not detected in one of the ears. A total of 10 ears 
(2.1\% of all ears) recorded no openings. In 3 ears, an insufficient number of openings was detected, i.e. one or two single openings. Similar results (1-2 of 10 openings) were obtained for all three different consecutive test maneuvers in these ears. No ET opening by water swallowing and 2 openings by dry swallowing were recorded in 1 subject only. In the other 7 ears, 7, 8, or 9 of $10 \mathrm{ET}$ openings were provoked by both dry and water swallowing.

Out of all not detected ET openings, almost half were reported during low-sip water maneuvers (49.17\%), 30\% occurred during large-sip water test maneuvers, while concealed ET openings during dry saliva swallowing test maneuvers constituted only $20.83 \%$.

The results obtained as related to the mean ET opening duration and the mean sound wave amplitude are presented in Table 1. The maximal and minimal opening duration and amplitude for various maneuvers are presented in Table 2. A comparison of different maneuvers showed no significant difference $(P>0.05)$. By form, the sonotubometry curves are summarized in Table 3. Examples of types of sonotubometry curves are presented in Fig. 2. Most recorded curves exhibited high and narrow spikes. In 73 individuals (69.5\%), the curve shape remained the same when different maneuvers were performed for both ears. In the other subjects, during different test maneuvers various curve shapes were produced, generally of 2 types and sometimes of three different types per subject. Curve shape did not

Table 1. Mean results of the sonotubometry for all test maneuvers $(P>0.05)$

\begin{tabular}{lcc}
\hline Test maneuver & $\begin{array}{c}\text { ET opening duration } \\
(\mathrm{ms})\end{array}$ & $\begin{array}{c}\text { Sound wave amplitude } \\
(\mathrm{dB})\end{array}$ \\
\hline Dry swallowing & $284 \pm 100$ & $13.90 \pm 6.74$ \\
2-mL Water swallowing & $263 \pm 93$ & $12.54 \pm 6.27$ \\
5-mL Water swallowing & $264 \pm 92$ & $13.38 \pm 6.39$ \\
Total average for all test & $270 \pm 96$ & $13.48 \pm 6.57$ \\
$\quad$ maneuvers & & \\
\hline
\end{tabular}

Values are presented as mean $\pm \mathrm{SD}$.

ET, Eustachian tube. depend on subject age, sex, test maneuver type, amount of water, nor the size of a sip $(P>0.05)$.

\section{DISCUSSION}

In recent years, the scientific literature is rich in experimental and clinical studies that analyze specific ET testing and new surgical treatment methods [20-25]. However, deficiency of criteria exists, which inhibits the objective diagnosis and assessment of ET dysfunction. In addition, there is no common consent for the definition of ET dysfunction, currently the term being used to describe inadequate ventilation of the middle ear. ET function is difficult to assess, and the testing applied is almost always subjective. It is imperative to adopt mutually acceptable diagnostic criteria to validate ET dysfunction.

For objective examination of the ET, manometric tests are performed, including forced response, pressure equilibration and inflation-deflation as main test maneuvers [8-10,26]. These methods are not considered physiological because they are carried out only under specific pathological conditions. In addition, measurement procedure may be complex and can result in discomfort for patients, which is especially relevant in examination of children.

Sonotubometry is one of the most valuable objective tests to diagnose ET function, yet it is not widespread or used in clinical application. The biggest advantages of this testing include its non-invasive nature, (which enables assessment of the function of the ET under physiological conditions [27]), its affordability, its suitability for both adults and children, and the absence of discomfort. However, the sonotubometry testing results are contradictory in the literature. During a credibility study of sonotubometric testing in healthy subjects, ET openings varied widely $[11,12,17,19]$. Having performed a sonotubometric test with pure sound frequences/pure sound signal stimuli, Mondain et al. [16] objectively detected only $62 \%$ openings, Virtanen [12]

Table 2. The maximal and minimal opening duration and amplitude for various test maneuvers $(P>0.05)$

\begin{tabular}{lccccc}
\hline \multirow{2}{*}{ Test maneuver } & \multicolumn{2}{c}{ ET opening duration $(\mathrm{ms})$} & & \multicolumn{2}{c}{ Sound wave amplitude $(\mathrm{dB})$} \\
\cline { 2 - 3 } \cline { 5 - 6 } & Range & Mean \pm SD & Range & Mean \pm SD \\
\hline Dry swallowing & $80-620$ & $284 \pm 100$ & & $5.01-38.93$ & $13,90 \pm 6.74$ \\
2-mL Water swallowing & $60-680$ & $263 \pm 93$ & $5.01-34.84$ & $12,54 \pm 6.27$ \\
5-mL Water swallowing & $100-700$ & $264 \pm 92$ & & $5.01-37,08$ & $13,38 \pm 6.39$ \\
\hline
\end{tabular}

ET, Eustachian tube.

Table 3. Different sonotubogram types associated with different test maneuvers $(P>0.05)$

\begin{tabular}{lcccc}
\hline \multirow{2}{*}{ Test maneuver } & \multicolumn{4}{c}{ Sonotubogram type (\%) } \\
\cline { 2 - 5 } & Spike & Double-peak & Descendant & Plateau \\
\hline Dry swallowing & 50.95 & 23.33 & 17.14 & 7.62 \\
2-mL water swallowing & 44.29 & 32.38 & 13.81 & 13.81 \\
5-mL water swallowing & 49.05 & 27.14 & 12.86 & 10.00 \\
Total average for all test maneuvers & 52.38 & 27.62 & 14.60 & 8.25 \\
\hline
\end{tabular}



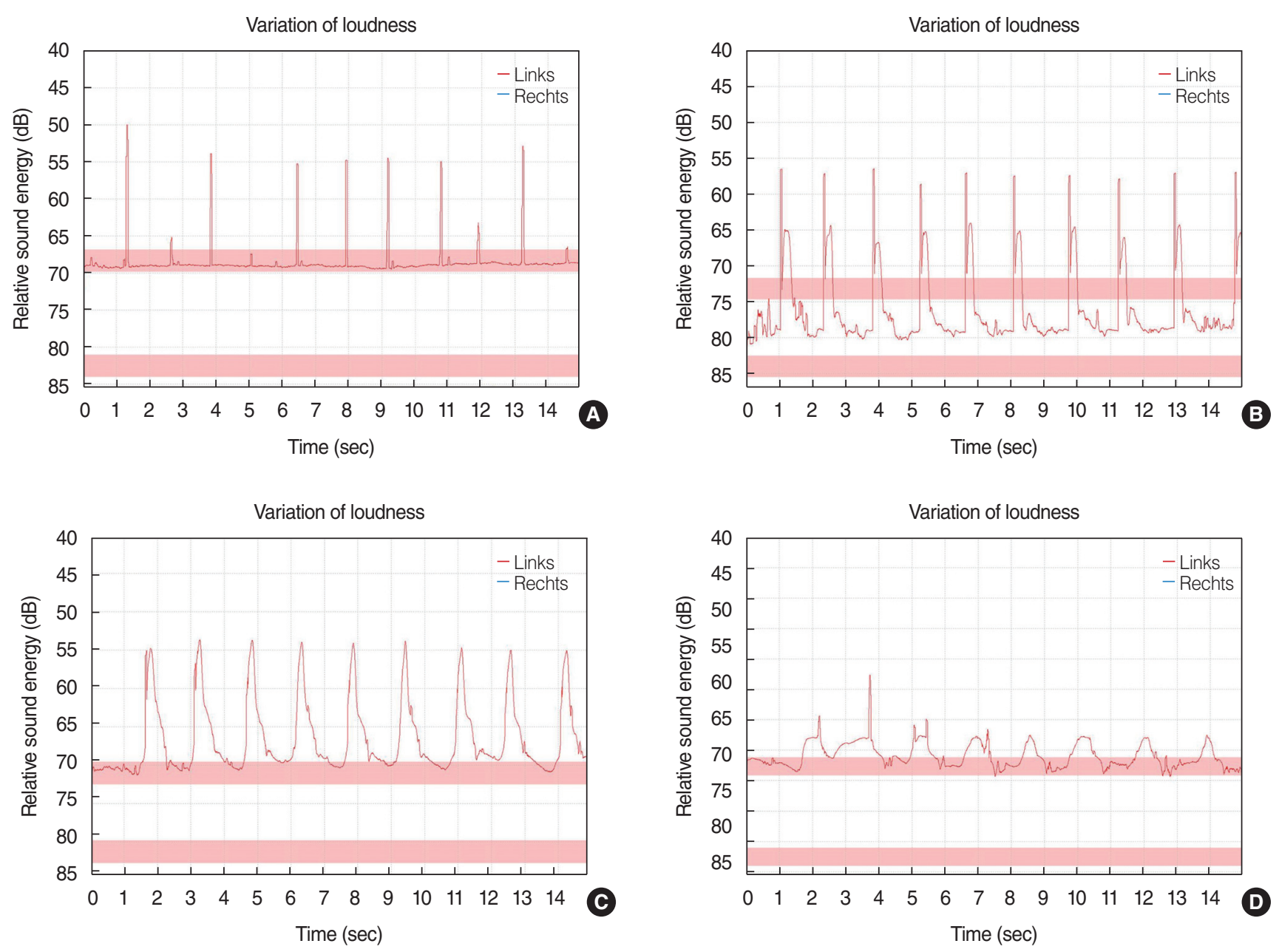

Fig. 2. Examples of types of sonotubometry curves. (A) Example for a spike-type sonotubogram. (B) Example for a double peak-type sonotubogram. (C) Example for a descendant-type sonotubogram. (D) Example for a plateau-type sonotubogram.

$90 \%$, Holmquist et al. [13] 66\%, Okubo et al. [14] 89\%, and Munro et al. [28] 80\%. Van der Avoort et al. [29,30] reported $81 \%$ ET openings in children and $91 \%$ in adults. This broad range of results obtained casts doubt on and questions the reliability of the approach with pure tone test signals.

Using the innovative PSEQ technology customized for sonotubometry contributes to more stable and reliable results (>90\%) [19]. During the PSEQ signal all frequency components $(0-48 \mathrm{kHz})$ are stimulated equally during every period, and this can provide a theoretical advantage to this signal. Using the PSEQ signal, Di Martino et al. [17,19] objectively recorded ET openings in almost all healthy subjects (but not in every action or maneuver), although the same group having performed a sonotubometric test with $8 \mathrm{-kHz}$ pure sound signal stimuli objectively detected only $55 \%$ of ET openings. Asenov et al. [18] detected at least one opening in $96.6 \%$ of measurements and $91.13 \%$ of tube openings in all measurements. Our current study succeeded in capturing ET openings in all subjects during almost all swallowings (98.1\%), using a greater number of sub- jects. Nonopenings were rare and did not depend on the type of test maneuver. It can be assumed that for healthy individuals with implicitly functional ET and no nose and nasopharynx pathology and where a high rate of ET openings occurs, the PSEQ signal technology increases the credibility of the testing and detects the number of ET openings quite objectively. Moreover, availability of the curve parameters enables quantification of the results. In order to better determine the specificity and sensitivity of the study, it is appropriate to compare the results of the sonotubometry for patients with nasal pathology, nasopharyngeal lymphoid hyperplasia or edema, and those with signs of ET functional impairment.

To have a technically simple and reliable test, it is important to standardize one test that induces ET openings and specific technique used. The key attribute of such a test is its reliability and maximum frequency of ET openings during multiple performances. An ET opening is induced by swallowing saliva, fluid or food and by yawning, and such tests as well as the Toynbee and Valsava maneuver are commonly used in sonotubometry. 
By comparing the interrelation of different maneuvers, complications to reproduce yawning and the Toynbee maneuver consecutively are distinguished. Another concern is that the ET opens relatively rarely-only in $40 \%-60 \%$ of cases-and these results are different even in the same subject. Saliva and water swallowing manuevers, nonetheless, are technically easier to perform and more reliable because almost every maneuver induces an ET opening. For that reason, conducting a number of consecutive tests reduces the influence of artefacts and generates reliable results [19]. In our current study for healthy adult individuals, we did not find an essential difference while detecting ET openings for 5 or 10 swallowing maneuvers. We aimed at discerning and gauging a reliable, simple and effective sonotubometry test with PSEQ, and we did not find any difference between the tests performed during dry or water swallowing. The amount of water and the size of a sip did not affect the sonotubometry result either. As comparison of different maneuvers showed no significant difference, any of them can be used. This standardized test deserves appropriate attention in further research relating to the study of dependency of middle ear diseases on ET function. Likewise, it is a rational solution in clinical practice where the efficient use of patient examination time is crucial and where the test performance must be easy and should exclude additional tools.

The quantitative parameters of the sonotubometric examination can be important while assessing different aspects of ET dysfunction, as curve parameters enable quantification of the results. There is not much data available about PSEQ stimuli based studies, and the assessment of the main PSEQ sonotubometry parameters indicates not exactly the same results in different tests. The parameters of the sound wave amplitude in open ET are similar and in healthy individuals they are $13-16 \mathrm{~dB}[18,19]$ (in current study, $13.48 \mathrm{~dB}$; SD, \pm 6.57 ), yet the results in the mean opening duration differ. In Di Martino et al. [19] PSEQ stimuli based study, a prolonged mean opening duration of the ET was registered (670 ms; SD, \pm 324 ), while in our current study the mean opening duration was shorter $(270 \mathrm{~ms}$; SD, \pm 96). Similar results were obtained in the study where healthy subjects and those with middle ear pathology were compared, since in healthy individuals the mean opening duration of the ET was $363 \mathrm{~ms}$ (SD, \pm 192 ), whereas in individuals with pathology it accounted for $280 \mathrm{~ms}(\mathrm{SD}, \pm 96)$ [18]. The difference in the results could be influenced either by the technical parameters of the sonotubometry device, or attributed to the study group profile.

The shape of the sonotubometry curve may determine the quality of the ET opening. In studies, different sonotubogram types are produced and they may indicate the quality or characteristics of the opening. Otherwise, these forms may be dependent on pharyngeal muscle activity patterns or the relationship between bone and cartilage in the ET, etc. However, so far the implication of the shape that a sonotubometry curve forms is unclear and requires further investigation. We noticed that certain sonotubogram types are relatively frequent $(52.38 \%$ spikeshaped curves and $27.62 \%$ double-peaked curves were recorded), while the other curves were erratic and less commonplace. In $69.5 \%$ of the subjects, the same curve shapes were reported in all maneuvers induced by dry or water swallowing, whereas the other individuals produced various curve shapes in different maneuvers. In order to determine causes affecting a curve shape, it would be reasonable to perform nasal videoendoscopy and to evaluate the ET opening mechanism, tubal dynamics, mucosal oedema, the amount of lymphoid tissue, pharyngeal muscle activity, etc. Further analysis of sonotubometry curves and other results in healthy and pathological cases can collect more data about the function of the ET and its assessment.

In conclusion, it is postulated that sonotubometry based on PSEQ stimuli is a reliable methodology to assess the ET opening function in healthy subjects. Mean ET opening duration time and the mean sound wave amplitude were similar performing all analyzed tests, hence it might be concluded that dry (saliva) and water swallowing are reliable sonotubometric maneuvers and may be used to examine ET opening function. Size of a sip during water swallowing does not affect the sonotubometry result. All maneuvers can be equally used as the optimal test maneuver, and we think that water swallowing is the most comfortable for the subject. Characteristics of sonotubometry performance (i.e., opening duration of the ET and the sound intensity variation) could be dependent on specific features of the sonotubometry device.

\section{CONFLICT OF INTEREST}

No potential conflict of interest relevant to this article was reported.

\section{REFERENCES}

1. Ockermann T, Reineke U, Upile T, Ebmeyer J, Sudhoff HH. Balloon dilation eustachian tuboplasty: a feasibility study. Otol Neurotol. 2010 Sep;31(7):1100-3.

2. Martino E, Di Thaden R, Krombach GA, Westhofen M. Function tests for the Eustachian tube: current knowledge. HNO. 2004 Nov; 52(11):1029-39.

3. Browning GG, Gatehouse S. The prevalence of middle ear disease in the adult British population. Clin Otolaryngol Allied Sci. 1992 Aug; 17(4):317-21.

4. McDonald MH, Hoffman MR, Gentry LR, Jiang JJ. New insights into mechanism of Eustachian tube ventilation based on cine computed tomography images. Eur Arch Otorhinolaryngol. 2012 Aug; 269(8):1901-7.

5. Bluestone CD. The Eustachian tube: structure, function, and role in the middle ear. Hamilton (CA): BC Decker Inc.; 2005.

6. Marchioni D, Mattioli F, Alicandri-Ciufelli M, Presutti L. Prevalence of ventilation blockages in patients affected by attic pathology: a 
case-control study. Laryngoscope. 2013 Nov;123(11):2845-53.

7. Leuwer R, Schubert R, Wenzel S, Kucinski T, Koch U, Maier H. New aspects of the mechanics of the auditory tube. HNO. 2003 May; 51(5):431-7.

8. van Heerbeek N, Ingels KJ, Snik AF, Zielhuis GA. Reliability of manometric eustachian tube function tests in children. Otol Neurotol. 2001 Mar;22(2):183-7.

9. Groth P, Ivarsson A, Tjernstrom O. Reliability in tests of the Eustachian tube function. Acta Otolaryngol. 1982;93(1-6):261-7.

10. Bluestone CD, Cantekin EI. Current clinical methods, indications and interpretation of eustachian tube function tests. Ann Otol Rhinol Laryngol. 1981 Nov-Dec;90(6 Pt 1):552-62.

11. van der Avoort SJ, van Heerbeek N, Zielhuis GA, Cremers CW. Sonotubometry: eustachian tube ventilatory function test. A state-ofthe-art review. Otol Neurotol. 2005 May;26(3):538-43.

12. Virtanen H. Sonotubometry: an acoustical method for objective measurement of auditory tubal opening. Acta Otolaryngol. 1978 Jul-Aug;86(1-2):93-103.

13. Holmquist J, Bjorkman G, Olen L. Measurement of eustachian tube function using sonotubometry. Scand Audiol. 1981 Feb;10(1):33-5.

14. Okubo J,Watanabe I, Shibusawa M, Ishikawa N, Ishida H,Teramura K. Sonotubometric measurement of the eustachian tube function by means of band noise: a clinical view of the acoustic measurement of the eustachian tube. ORL J Otorhinolaryngol Relat Spec. 1987 Feb; 49(5):242-52.

15. Jonathan $\mathrm{D}$. The predictive value of eustachian tube function (measured with sonotubometry) in the successful outcome of myringoplasty. Clin Otolaryngol Allied Sci. 1990 Oct;15(5):431-4.

16. Mondain M, Vidal D, Bouhanna S, Uziel A. Monitoring eustachian tube opening: preliminary results in normal subjects. Laryngoscope. 1997 Oct;107(10):1414-9.

17. Di Martino EF, Thaden R, Antweiler C, Reineke T, Westhofen M, Beckschebe J, et al. Evaluation of Eustachian tube function by sonotubometry: results and reliability of $8 \mathrm{kHz}$ signals in normal subjects. Eur Arch Otorhinolaryngol. 2007 Mar;264(3):231-6.

18. Asenov DR, Nath V, Telle A, Antweiler C, Walther LE, Vary P, et al. Sonotubometry with perfect sequences: first results in pathological ears. Acta Otolaryngol. 2010 Nov;130(11):1242-8.

19. Di Martino EF, Nath V, Telle A, Antweiler C, Walther LE, Vary P. Evaluation of Eustachian tube function with perfect sequences: technical realization and first clinical results. Eur Arch Otorhinolaryngol. 2010 Mar;267(3):367-74.

20. Poe DS, Abou-Halawa A, Abdel-Razek O. Analysis of the dysfunctional eustachian tube by video endoscopy. Otol Neurotol. 2001 Sep;22(5):590-5.

21. Poe DS, Grimmer JF, Metson R. Laser eustachian tuboplasty: twoyear results. Laryngoscope. 2007 Feb;117(2):231-7.

22. Schloegel L, Gottschall JA. Balloon dilation of the Eustachian tube: safety and utility. Otolaryngol Head Neck Surg. 2009 Sep;141(3S1): 134.

23. Poe DS, Silvola J, Pyykko I. Balloon dilation of the cartilaginous Eustachian tube. Otolaryngol Head Neck Surg. 2011Apr;144(4):563-9.

24. Takasaki K, Sando I, Balaban CD, Miura M. Functional anatomy of the tensor veli palatini muscle and Ostmann's fatty tissue. Ann Otol Rhinol Laryngol. 2002 Nov;111(11):1045-9.

25. Gaihede M, Dirckx JJ, Jacobsen H,Aernouts J, Sovso M,Tveteras K. Middle ear pressure regulation: complementary active actions of the mastoid and the Eustachian tube. Otol Neurotol. 2010 Jun;31(4): 603-11.

26. Bylander A,Tjernstrom O, Ivarsson A. Pressure opening and closing functions of the eustachian tube in children and adults with normal ears. Acta Otolaryngol. 1983 Jan-Feb;95(1-2):55-62.

27. Honjo I. Eustachian tube in middle ear diseases. New York: Springer; 1988.

28. Munro KJ, Benton CL, Marchbanks RJ. Sonotubometry findings in children at high risk from middle ear effusion. Clin Otolaryngol Allied Sci. 1999 Jun;24(3):223-7.

29. van der Avoort SJ, Heerbeek Nv, Zielhuis GA, Cremers CW.Validation of sonotubometry in healthy adults. J Laryngol Otol. 2006 Oct; 120(10):853-6.

30. van der Avoort SJ, van Heerbeek N, Snik AF, Zielhuis GA, Cremers CW. Reproducibility of sonotubometry as Eustachian tube ventilatory function test in healthy children. Int J Pediatr Otorhinolaryngol. 2007 Feb;71(2):291-5. 